\title{
A new traveltime approximation for TI media
}

\author{
Alexey Stovas ${ }^{1}$ and Tariq Alkhalifah ${ }^{2}$
}

\begin{abstract}
In a transversely isotropic (TI) medium, the trade-off between inhomogeneity and anisotropy can dramatically reduce our capability to estimate anisotropy parameters. By expanding the TI eikonal equation in power series in terms of the aneliptic parameter $\eta$, we derive an efficient tool to estimate (scan) for $\eta$ in a generally inhomogeneous, elliptically anisotropic background medium. For a homogeneoustilted transversely isotropic medium, we obtain an analytic nonhyperbolic moveout equation that is accurate for large offsets. In the common case where we do not have well information and it is necessary to resolve the vertical velocity, the background medium can be assumed isotropic, and the traveltime equations becomes simpler. In all cases, the accuracy of this new TI traveltime equation exceeds previously published formulations and demonstrates how $\eta$ is better resolved at small offsets when the tilt is large.
\end{abstract}

\section{INTRODUCTION}

A transversely isotropic (TI) model with a tilted symmetry axis is one of the most effective approximations to the earth subsurface, especially for imaging purposes (Zhou et al., 2004 and Huang et al., 2008). In fact, constraining the symmetry direction angle to be normal to the reflector dip allows us to mitigate a lot of the uncertainty in parameter estimation (Audubert et al., 2006 and Alkhalifah and Sava, 2010). Simplifying traveltime formulation for such a model helps in many applications, including traveltime tomography and integral-based Kirchhoff migration. The P-wave traveltimes in 2D TI medium are dependent on the parameters in symmetry direction, the on-axis velocity $v_{0}$, the on-axis normalmoveout velocity $v_{n m o}=v_{0} \sqrt{1+2 \delta}$, where $\delta$ is the anisotropy parameter and the on-axis anellipticity parameter is $\eta$. The fourth parameter is the symmetry direction angle.

To find a stable solution for the TI eikonal equation using finitedifference schemes is generally a hard task, especially because such a process requires finding the root of a quartic equation (or higher order for the tilted case) at each computational step. However, traveltime computation for a simplified, but not practical, elliptically anisotropic model is far more efficient, even if the symmetry axis is tilted. Like the isotropic case, such an elliptical eikonal equation solution requires solving a quadratic equation at each computational step, which is trivial and straightforward. Having such a model as a launching pad for our perturbation in $\eta$ provides us an opportunity to calculate traveltimes for TTI media in a generally inhomogeneous background model.

Alkhalifah (2011a) used perturbation theory to develop traveltime solutions for a transversely isotropic media with a vertical axis of symmetry (VTI). However, unlike Alkhalifah (2011b), who continued to use a vertical elliptical isotropy background medium, and thus had to perturb the tilt angle and $\eta$, we use a tilted elliptical anisotropic background model for our perturbation expansion, which allows us to focus on expansions in $\eta$ only. The result is higher accuracy and less uncertainty, especially if we fix the tilt direction to be normal to the reflector dip, as is the practice of the industry in many areas. Unlike Alkhalifah (2011b), the symmetry axis is allowed to vary, because it is part of the background tilted elliptical isotropic model. In this case, the traveltime can be estimated using the associated eikonal (or ray-tracing) equations.

\section{THE EXPANSIONS OF TI EIKONAL}

The 2D eikonal equation in VTI media is given by (Alkhalifah, 1998)

$$
v_{n m o}^{2}(1+2 \eta)\left(\frac{\partial \tau}{\partial x}\right)^{2}+v_{0}^{2}\left(\frac{\partial \tau}{\partial z}\right)^{2}\left(1-2 \eta v_{n m o}^{2}\left(\frac{\partial \tau}{\partial x}\right)^{2}\right)=1
$$

\footnotetext{
Manuscript received by the Editor 3 May 2011; revised manuscript received 14 November 2011; published online 6 July 2012.

${ }^{1}$ Norwegian University of Science and Technology (NTNU), Department of Petroleum Engineering and Applied Geophysics, Trondheim, Norway. E-mail: alexey@ntnu.no.

${ }^{2}$ King Abdullah University for Science and Technology (KAUST), Physical Science Division, Saudi Arabia. E-mail: tariq.alkhalifah@kaust.edu.sa. (C) 2012 Society of Exploration Geophysicists. All rights reserved.
} 
where $\tau(x, z)$ is the traveltime measured from the source to a receiver point $(x, z), v_{0}$ and $v_{n m o}$ are the vertical and NMO velocities measured along the axis of symmetry (vertical axis in the VTI case) and $\eta$ is the anellipticity parameter. In perturbing the eikonal equation, all parameters related to the background medium are allowed to vary freely with position (inhomogeneous), whereas the perturbation parameters are considered constant. In the following, we review the development of Alkhalifah (2011b), which was based on perturbing from a background medium with a vertically symmetry axis anisotropy and thus always required a constant symmetry axis.

For a tilted 2D TI medium, we have the rotation operator

$$
\left(\begin{array}{cc}
\cos \theta & \sin \theta \\
-\sin \theta & \cos \theta
\end{array}\right),
$$

where $\theta$ is the angle that the symmetry axis makes with the vertical. The 2D eikonal equation in TTI media takes the form (Alkhalifah, 2011b)

$$
\begin{aligned}
& v_{n m o}^{2}(1+2 \eta)\left(\cos \theta \frac{\partial \tau}{\partial x}+\sin \theta \frac{\partial \tau}{\partial z}\right)^{2} \\
& +v_{0}^{2}\left(\cos \theta \frac{\partial \tau}{\partial z}-\sin \theta \frac{\partial \tau}{\partial x}\right)^{2} \\
& \quad \times\left(1-2 \eta v_{n m o}^{2}\left(\cos \theta \frac{\partial \tau}{\partial x}+\sin \theta \frac{\partial \tau}{\partial z}\right)^{2}\right)=1
\end{aligned}
$$

The full 3D version of equation 3 can be found in (Alkhalifah, 2011b, Appendix D).

Solving equation 3 numerically requires solving a quartic equation (see Alkhalifah, 2011b).

Alkhalifah (2011b) proposed to use one- and two-parameter expansions as the trial solution of equation 3. The one-parameter expansion

$$
\tau(x, z) \approx \tau_{0}(x, z)+\tau_{1}(x, z) \sin \theta+\tau_{2}(x, z) \sin ^{2} \theta
$$

with VTI background medium, where $\tau_{0}(x, z)$ satisfies the eikonal equation for VTI medium (equation 1 ), while $\tau_{i}(x, z), i=1,2$ satisfy the linear first-order partial differential equation

$$
\begin{aligned}
& v_{n m o}^{2}(1+2 \eta) \frac{\partial \tau_{0}}{\partial x} \frac{\partial \tau_{i}}{\partial x}+v_{0}^{2} \frac{\partial \tau_{0}}{\partial z} \frac{\partial \tau_{i}}{\partial z}-2 \eta \\
& v_{n m o}^{2} v_{0}^{2} \frac{\partial \tau_{0}}{\partial z} \frac{\partial \tau_{0}}{\partial x}\left(\frac{\partial \tau_{0}}{\partial z} \frac{\partial \tau_{i}}{\partial x}+\frac{\partial \tau_{0}}{\partial x} \frac{\partial \tau_{i}}{\partial z}\right)=f_{i}(x, z) \\
& i=1,2
\end{aligned}
$$

where the right side $f_{i}(x, z)$ depends on terms that can be evaluated sequentially (Alkhalifah, 2011b, Appendix A).

The two-parameter expansion

$$
\begin{gathered}
\tau(x, z) \approx \tau_{0}(x, z)+\tau_{\eta}(x, z) \eta+\tau_{\theta}(x, z) \sin \theta+ \\
\tau_{\eta 2}(x, z) \eta^{2}+\tau_{\eta \theta}(x, z) \eta \sin \theta+\tau_{\theta 2}(x, z) \sin ^{2} \theta
\end{gathered}
$$

is elliptically isotropic, with a vertical symmetry direction background. Substituting equation 6 into equation 1 results in the following system of linear first-order partial differential equations:

$$
v_{n m o}^{2} \frac{\partial \tau_{0}}{\partial x} \frac{\partial \tau_{i}}{\partial x}+v_{0}^{2} \frac{\partial \tau_{0}}{\partial z} \frac{\partial \tau_{i}}{\partial z}=f_{i}(x, z), \quad i=\eta, \theta, \eta_{2}, \eta \theta, \theta_{2}
$$

where $\tau_{0}(x, z)$ satisfies the eikonal equation for an elliptical anisotropic background model by setting $\eta=0$ in equation 1

$$
v_{n m o}^{2}\left(\frac{\partial \tau}{\partial x}\right)^{2}+v_{0}^{2}\left(\frac{\partial \tau}{\partial z}\right)^{2}=1
$$

\section{THE TI EIKONAL EQUATION}

Here, we propose to use a tilted elliptically isotropic medium (TEI) as a background model and perform expansion in the parameter $\eta$ only.

The $2 \mathrm{D}$ eikonal equation in TEI media takes the form (by setting $\eta=0$ in equation 3 )

$$
\begin{aligned}
& v_{\text {nmo }}^{2}\left(\cos \theta \frac{\partial \tau}{\partial x}+\sin \theta \frac{\partial \tau}{\partial z}\right)^{2} \\
& +v_{0}^{2}\left(\cos \theta \frac{\partial \tau}{\partial z}-\sin \theta \frac{\partial \tau}{\partial x}\right)^{2}=1
\end{aligned}
$$

We can use the following trial solution, similar to Alkhalifah (2011a), and assume $\eta$ to be constant and independent,

$$
\tau(x, z) \approx \tau_{0}(x, z)+\tau_{1}(x, z) \eta+\tau_{2}(x, z) \eta^{2} .
$$

The required functions $\tau_{i}(x, z), i=1,2$ can be found using the same scheme as in Alkhalifah (2011b). The coefficient of firstpower in $\eta$ can be found from

$$
\begin{aligned}
& \left(v_{n m o}^{2} \cos ^{2} \theta+v_{0}^{2} \sin ^{2} \theta\right) \frac{\partial \tau_{0}}{\partial x} \\
& \left.+\sin \theta \cos \theta\left(v_{n m o}^{2}-v_{0}^{2}\right) \frac{\partial \tau_{0}}{\partial z}\right) \frac{\partial \tau_{1}}{\partial x} \\
& +\left(\sin \theta \cos \theta\left(v_{n m o}^{2}-v_{0}^{2}\right) \frac{\partial \tau_{0}}{\partial x}\right. \\
& \left.+\left(v_{n m o}^{2} \sin ^{2} \theta+v_{0}^{2} \cos ^{2} \theta\right) \frac{\partial \tau_{0}}{\partial z}\right) \frac{\partial \tau_{1}}{\partial z} \\
& \quad=f_{1}\left(\frac{\partial \tau_{0}}{\partial x}, \frac{\partial \tau_{0}}{\partial z}\right),
\end{aligned}
$$

where the right side is the function of the zero-power coefficient $\tau_{0}(x, z)$

$$
\begin{aligned}
f_{1}\left(\frac{\partial \tau_{0}}{\partial x}, \frac{\partial \tau_{0}}{\partial z}\right)= & -v_{n m o}^{2}\left(\cos \theta \frac{\partial \tau_{0}}{\partial x}+\sin \theta \frac{\partial \tau_{0}}{\partial z}\right)^{2} \\
& +v_{n m o}^{2} v_{0}^{2}\left(-\sin \theta \frac{\partial \tau_{0}}{\partial x}+\cos \theta \frac{\partial \tau_{0}}{\partial z}\right)^{2} \\
& \times\left(\cos \theta \frac{\partial \tau_{0}}{\partial x}+\sin \theta \frac{\partial \tau_{0}}{\partial z}\right)^{2}
\end{aligned}
$$


The coefficient of second-power in $\eta$ can be found from

$$
\begin{aligned}
& 2\left(\left(v_{n m o}^{2} \cos ^{2} \theta+v_{0}^{2} \sin ^{2} \theta\right) \frac{\partial \tau_{0}}{\partial x}\right. \\
& \left.+\sin \theta \cos \theta\left(v_{n m o}^{2}-v_{0}^{2}\right)\right) \frac{\partial \tau_{2}}{\partial x} \\
& +2\left(\sin \theta \cos \theta\left(v_{n m o}^{2}-v_{0}^{2}\right) \frac{\partial \tau_{0}}{\partial x}\right. \\
& \left.+\left(v_{n m o}^{2} \sin ^{2} \theta+v_{0}^{2} \cos ^{2} \theta\right)\right) \frac{\partial \tau_{2}}{\partial z} \\
& \quad=f_{2}\left(\frac{\partial \tau_{0}}{\partial x}, \frac{\partial \tau_{0}}{\partial z}, \frac{\partial \tau_{1}}{\partial x}, \frac{\partial \tau_{1}}{\partial z}\right),
\end{aligned}
$$

where the right side is the function of the zero-power coefficient $\tau_{0}(x, z)$ and the first-power coefficient $\tau_{1}(x, z)$

$$
\begin{aligned}
& f_{2}\left(\frac{\partial \tau_{0}}{\partial x}, \frac{\partial \tau_{0}}{\partial z}, \frac{\partial \tau_{1}}{\partial x}, \frac{\partial \tau_{1}}{\partial z}\right)=-v_{0}^{2}\left(-\sin \theta \frac{\partial \tau_{1}}{\partial x}+\cos \theta \frac{\partial \tau_{1}}{\partial z}\right)^{2} \\
& \quad-v_{n m o}^{2}\left(\left(\cos \theta \frac{\partial \tau_{1}}{\partial x}+\sin \theta \frac{\partial \tau_{1}}{\partial z}\right)^{2}\right. \\
& \left.\quad+4\left(\cos \theta \frac{\partial \tau_{0}}{\partial x}+\sin \theta \frac{\partial \tau_{0}}{\partial z}\right)\left(\cos \theta \frac{\partial \tau_{1}}{\partial x}+\sin \theta \frac{\partial \tau_{1}}{\partial z}\right)\right) \\
& \quad+4 v_{0}^{2} v_{n m o}^{2}\left(\left(-\sin \theta \frac{\partial \tau_{0}}{\partial x}+\cos \theta \frac{\partial \tau_{0}}{\partial z}\right)\right. \\
& \quad \times\left(\cos \theta \frac{\partial \tau_{0}}{\partial x}+\sin \theta \frac{\partial \tau_{0}}{\partial z}\right)^{2}\left(-\sin \theta \frac{\partial \tau_{1}}{\partial x}+\cos \theta \frac{\partial \tau_{1}}{\partial z}\right) \\
& \quad+\left(-\sin \theta \frac{\partial \tau_{0}}{\partial x}+\cos \theta \frac{\partial \tau_{0}}{\partial z}\right)^{2}\left(\cos \theta \frac{\partial \tau_{0}}{\partial x}+\sin \theta \frac{\partial \tau_{0}}{\partial z}\right) \\
& \left.\quad \times\left(\cos \theta \frac{\partial \tau_{1}}{\partial x}+\sin \theta \frac{\partial \tau_{1}}{\partial z}\right)\right)
\end{aligned}
$$

\section{A HOMOGENEOUS MODEL TEST}

Although the above equation can provide travelimes corresponding to an inhomogeneous background medium allowing the velocities and the symmetry axis to vary, we test the accuracy for a homogenous medium because we can easily compute the exact traveltimes for this case. Thus, we define the vertical and NMO velocities in the vertical direction for a tilted elliptically isotropic medium, $V_{0}$ and $V_{n m o}$, respectively, following Golikov and Stovas (2011)

$$
V_{0}^{2}=v_{0}^{2} \cos ^{2} \theta+v_{n m o}^{2} \sin ^{2} \theta \quad \frac{1}{V_{n m o}^{2}}=\frac{\cos ^{2} \theta}{v_{n m o}^{2}}+\frac{\sin ^{2} \theta}{v_{0}^{2}}
$$

with inverse transform

$$
\begin{gathered}
v_{0}^{2}=\frac{1}{2 \cos ^{2} \theta}\left(V_{0}^{2}+\sqrt{V_{0}^{4}-V_{n m o}^{2} V_{0}^{2} \sin ^{2} 2 \theta}\right) \\
\frac{1}{v_{n m o}^{2}}=\frac{1}{2 \cos ^{2} \theta}\left(\frac{1}{V_{n m o}^{2}}+\sqrt{\frac{1}{V_{n m o}^{4}}-\frac{\sin ^{2} 2 \theta}{V_{n m o}^{2} V_{0}^{2}}}\right) .
\end{gathered}
$$

The product of vertical and NMO velocities is tilt-independent (Golikov and Stovas, 2011), i.e.,

$$
v_{0}^{2} v_{n m o}^{2}=V_{0}^{2} V_{n m o}^{2}
$$

Next, we solve equation 9 for a tilted elliptically isotropic medium that we choose as a background medium. Notice that in a homogeneous-tilted elliptically isotropic medium, the traveltime equation is hyperbolic with shift in the lateral variable,

$$
\begin{aligned}
\tau_{0}(x, z) & =\sqrt{\frac{\left(x-x_{0}\right)^{2}}{V_{n m o}^{2}}+\frac{z^{2}}{V_{0}^{2}}} \\
& =\sqrt{\frac{(x \cos \theta+z \sin \theta)^{2}}{v_{n m o}^{2}}+\frac{(-x \sin \theta+z \cos \theta)^{2}}{v_{0}^{2}}},
\end{aligned}
$$

where the shift $x_{0}$ is given by (Golikov and Stovas, 2011)

$$
\begin{aligned}
x_{0} & =\frac{z\left(v_{n m o}^{2}-v_{0}^{2}\right) \sin \theta \cos \theta}{v_{0}^{2} \cos ^{2} \theta+v_{n m o}^{2} \sin ^{2} \theta} \\
& =\frac{z \sin 2 \theta\left(V_{n m o}^{2}-V_{0}^{2}\right)}{V_{n m o}^{2}\left(\cos 2 \theta+\sqrt{1-\frac{V_{n m o}^{2}}{V_{0}^{2}} \sin ^{2} 2 \theta}\right)} .
\end{aligned}
$$

Substituting equation 19 into equation 18 and expanding in a series of sines of tilt truncated after the second-order term, we obtain equation 8 from Alkhalifah (2011b).

Now equation 18 can be used as $\tau_{0}(x, z)$ in trial solution 10 . The main advantage of using equation 18 instead of equation 8 in Alkhalifah (2011b) is more accurate description of the eikonal equation solution for near-vertical propagation.

Substituting equation 18 into equation 12 gives

$$
f_{1}\left(\frac{\partial \tau_{0}}{\partial x}, \frac{\partial \tau_{0}}{\partial z}\right)=-\frac{v_{0}^{4}(x \cos \theta+z \sin \theta)^{4}}{\left(v_{0}^{2}(x \cos \theta+z \sin \theta)^{2}+v_{\text {nmo }}^{2}(-x \sin \theta+z \cos \theta)^{2}\right)^{2}} .
$$

Substituting equation 20 into equation 11 results in the firstpower coefficient

$$
\tau_{1}(x, z)=-\frac{v_{0}^{4}(x \cos \theta+z \sin \theta)^{4} \sqrt{\frac{(x \cos \theta+z \sin \theta)^{2}}{v_{n m o}^{2}}+\frac{(-x \sin \theta+z \cos \theta)^{2}}{v_{0}^{2}}}}{\left(v_{0}^{2}(x \cos \theta+z \sin \theta)^{2}+v_{n m o}^{2}(-x \sin \theta+z \cos \theta)^{2}\right)^{2}} .
$$

The second-power coefficient can be found in similar way, 


$$
\begin{aligned}
\tau_{2}(x, z)= & \frac{3 v_{0}^{6}(x \cos \theta+z \sin \theta)^{6}\left(v_{0}^{2}(x \cos \theta+z \sin \theta)^{2}+4 v_{n m o}^{2}(-x \sin \theta+z \cos \theta)^{2}\right)}{2\left(v_{0}^{2}(x \cos \theta+z \sin \theta)^{2}+v_{n m o}^{2}(-x \sin \theta+z \cos \theta)^{2}\right)^{4}} * \\
& \sqrt{\frac{(x \cos \theta+z \sin \theta)^{2}}{v_{n m o}^{2}}+\frac{(-x \sin \theta+z \cos \theta)^{2}}{v_{0}^{2}}}
\end{aligned}
$$

Substituting equations 18,21 , and 22 into the trial solution 10 results in the following eikonal equation solution approximation

$$
\begin{aligned}
\tau(x, z) & =\sqrt{\frac{(x \cos \theta+z \sin \theta)^{2}}{v_{n m o}^{2}}+\frac{(-x \sin \theta+z \cos \theta)^{2}}{v_{0}^{2}}} \\
& \times\left(1-\frac{v_{0}^{4}(x \cos \theta+z \sin \theta)^{4} \eta}{\left(v_{0}^{2}(x \cos \theta+z \sin \theta)^{2}+v_{n m o}^{2}(-x \sin \theta+z \cos \theta)^{2}\right)^{2}}\right. \\
& \left.+\frac{3 v_{0}^{6}(x \cos \theta+z \sin \theta)^{6}\left(v_{0}^{2}(x \cos \theta+z \sin \theta)^{2}+4 v_{n m o}^{2}(-x \sin \theta+z \cos \theta)^{2}\right) \eta^{2}}{2\left(v_{0}^{2}(x \cos \theta+z \sin \theta)^{2}+v_{n m o}^{2}(-x \sin \theta+z \cos \theta)^{2}\right)^{4}}\right) .
\end{aligned}
$$

As soon as coefficients $\tau_{1}$ and $\tau_{2}$ are computed, they can be used, as is shown by Alkhalifah (2011b), to estimate the traveltime using the first-sequence of Shanks transform (Bender and Orszag, 1978)

$$
\tau(x, z) \approx \frac{A_{0} A_{2}-A_{1}^{2}}{A_{0}-2 A_{1}+A_{2}}
$$

where

$$
A_{0}=\tau_{0} \quad A_{1}=\tau_{0}+\tau_{1} \eta \quad A_{2}=\tau_{0}+\tau_{1} \eta+\tau_{2} \eta^{2} .
$$

This transform tends to predict the behavior of the higher (eliminated) order terms of the sequence, and thus improve the accuracy of the expansion (Bender and Orszag, 1978). By substituting expressions for $\tau_{0}, \tau_{1}$ and $\tau_{2}$ into equation 25 and consequently into equation 24 we obtain

$$
\begin{aligned}
& \tau(x, z) \\
& \quad \approx \sqrt{\frac{(x \cos \theta+z \sin \theta)^{2}}{v_{n m o}^{2}}+\frac{(-x \sin \theta+z \cos \theta)^{2}}{v_{0}^{2}}} \frac{1+\Phi_{1}(x, z) \eta}{1+\Phi_{2}(x, z) \eta},
\end{aligned}
$$

where

$$
\begin{gathered}
\Phi_{1}(x, z)=\frac{v_{0}^{2}(x \cos \theta+z \sin \theta)^{2}}{\left(v_{0}^{2}(x \cos \theta+z \sin \theta)^{2}+v_{n m o}^{2}(-x \sin \theta+z \cos \theta)^{2}\right)^{2}} \\
*\left(\frac{1}{2} v_{0}^{2}(x \cos \theta+z \sin \theta)^{2}+6 v_{n m o}^{2}(-x \sin \theta+z \cos \theta)^{2}\right) \\
\Phi_{2}(x, z)=\frac{3 v_{0}^{2}(x \cos \theta+z \sin \theta)^{2}}{2\left(v_{0}^{2}(x \cos \theta+z \sin \theta)^{2}+v_{n m o}^{2}(-x \sin \theta+z \cos \theta)^{2}\right)^{2}} \\
*\left(v_{0}^{2}(x \cos \theta+z \sin \theta)^{2}+4 v_{n m o}^{2}(-x \sin \theta+z \cos \theta)^{2}\right) .
\end{gathered}
$$

We can also consider the very practical special case with $v_{n m o}=$ $v_{0}$ or $\delta=0$, often considered in the absence of well or other a priori information to resolve the vertical velocity. The traveltime equation 18 for a background medium reduces to the isotropic traveltime equation

$$
\tau_{0}(x, z)=\frac{1}{v_{0}} \sqrt{x^{2}+z^{2}}
$$

while the first- and second-order coefficients are simplified to

$$
\tau_{1}(x, z)=-\frac{(x \cos \theta+z \sin \theta)^{4}}{v_{0}\left(x^{2}+z^{2}\right)^{3 / 2}}
$$

$\tau_{2}(x, z)=\frac{3(x \cos \theta+z \sin \theta)^{6}\left(x^{2}\left(1+3 \sin ^{2} \theta\right)-6 x z \sin \theta \cos \theta+z^{2}\left(4-3 \sin ^{2} \theta\right)\right)}{2 v_{0}\left(x^{2}+z^{2}\right)^{7 / 2}}$.

Note that the expansion with coefficients defined in equations 28 30 is different from the one proposed by Alkhalifah (2011b) (see equation 6). Note in equation 29 that this first-order coefficient for $\eta$ increases with offset, but the influence of offset decreases with increasing tilt in the symmetry axis. Thus, there is a tradeoff between offset and symmetry angle in the first-order coefficient of the $\eta$ influence, where, for a large tilt in the symmetry axis, the influence of $\eta$ does not require large offsets.

\section{NUMERICAL TESTS}

For comparison, we use approximations developed in Pech et al. (2003), Grechka and Pech (2006), Sena (1991), and the homogeneous background medium version of the perturbation approach developed by Alkhalifah (2011b) for an elliptically isotropic background medium with a vertical symmetry axis. To test the accuracy of moveout approximations, we select a homogeneous TTI medium with a reflector depth of $z=2 \mathrm{~km}$, axis-direction velocity $v_{0}=2 \mathrm{~km} / \mathrm{s}$, anisotropic parameters $\delta=0.0, \eta=0.2$, and tilt $\theta=$ $20^{\circ}$ (model one). The results are shown in Figure 1. One can see that the proposed approximation is superior in accuracy for small and large offset. The results computed for similar model with anisotropic parameters $\delta=0.1, \eta=0.2$, and tilt $\theta=20^{\circ}$ (model two) are shown in Figure 1, right.

In Figure 2, we show the relative error in traveltime computed using the zero-, first-, and second-order approximations from equation 10 and the Shanks transformation given in equations 26-27 for model two with different symmetry axis direction. One can see that the Shanks transform is superior in accuracy regardless to the symmetry axis direction. The zero-error corresponds to offset obtained from on-symmetry axis point at the slowness surface $(x=z \tan \theta)$ that does not depend on $\eta$. This Figure clearly demonstrates the effectiveness of the Shanks transform in improving the accuracy of the expansion.

There is a general tradeoff between $\eta$ and the symmetry angle that may require some a priori information for $\eta$ or constraining the tilt to be normal to the reflector dip. Figure 3 shows the difference in traveltime computed from equations 18 and 23 (corresponding to tilted elliptically isotropic and TTI media, respectively) for model two and different tilts, which demonstrates that the influence and resolution of $\eta$ takes place at large offsets for small tilt angles. However, as the tilt increases, the resolution, for tomography type inversions, increases, but the change with offset, which may be critical for semblance type estimations, decreases.

Figure 4 shows the percent error in the vertical traveltime (top) and the vertical NMO velocity (bottom) extracted through linearization of the new equation for a $20^{\circ}$ tilt angle (left) and $40^{\circ}$ tilt angle (right). The accuracy of the new formulation for reasonable values of $\eta$ is high for the vertical traveltime and 
a)

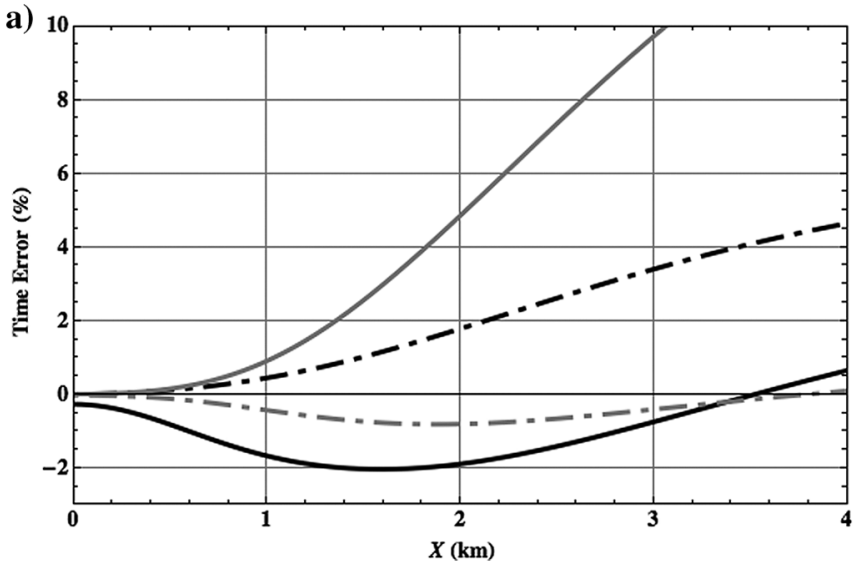

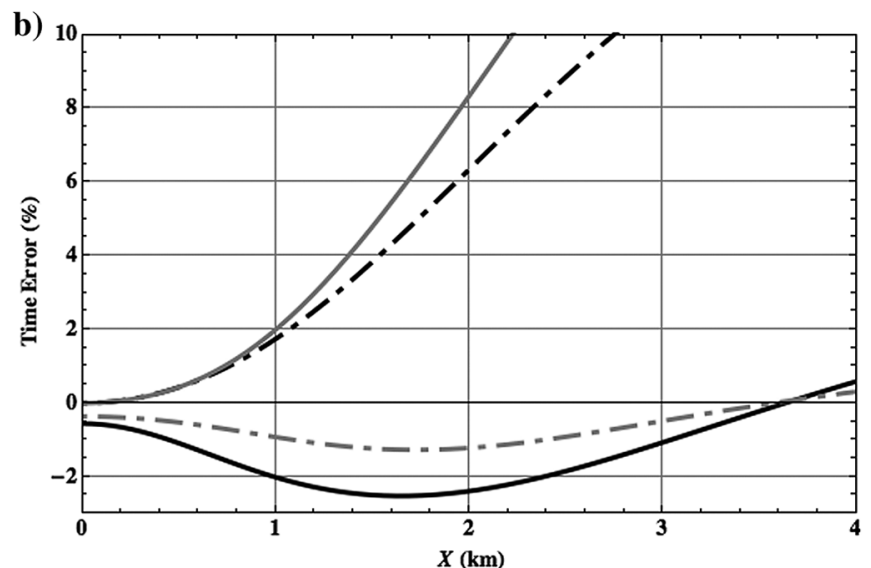

Figure 1. Relative error in traveltime computed from different approximations for model one (left) and model two (right). Grechka and Pech (2006) — solid gray curve, Sena (1991) — dashed black curve, Alkhalifah (2011b) — solid black curve, and the proposed approximation - dashed gray curve.

NMO velocity. As the tilt increases, the error in the vertical NMO velocity only is dependent on $\eta$.

\section{DISCUSSIONS}

Despite using the new perturbation-based partial differential equations for TTI traveltimes to derive analytical formulas for homogeneous media, the real value of the new formulation is in its capability in predicting traveltimes in inhomogeneous media. If we manage to derive an inhomogeneous velocity model from, for example, isotropic migration velocity analysis, we can use that velocity along with an assumption on the symmetry axis direction (for example, in the direction of the velocity gradient) to calculate $\tau_{0}(x, z)$, followed by $\tau_{1}(x, z)$ and then $\tau_{2}(x, z)$, the coefficients of the $\eta$ expansion. Using these coefficients, we can, based on equation 24 , estimate traveltimes analytically for any constant $\eta$ in this inhomogeneous background medium. We also can scan for $\eta$ values that provide traveltimes that fit the measured ones from, for example, picked seismic arrivals. Though the theoretical development assumes constant $\eta$, we can allow $\eta$ to vary smoothly as an effective parameter. The relation between these effective values and the interval ones depends a lot on the inhomogeneity of the background medium.

Alkhalifah (2011a) demonstrated the accuracy of such perturbation-based solution for a special case of TTI called VTI. Because the TTI case eikonal is a rotation version of the VTI one, the accuracy of the perturbation-based solution for an inhomogeneous background is expected to be the same. Nevertheless, the approach here suggested a more practical framework in dealing with TTI than the approach suggested by Alkhalifah (2011b) based in a vertical elliptical anisotropic background. It also resulted in more
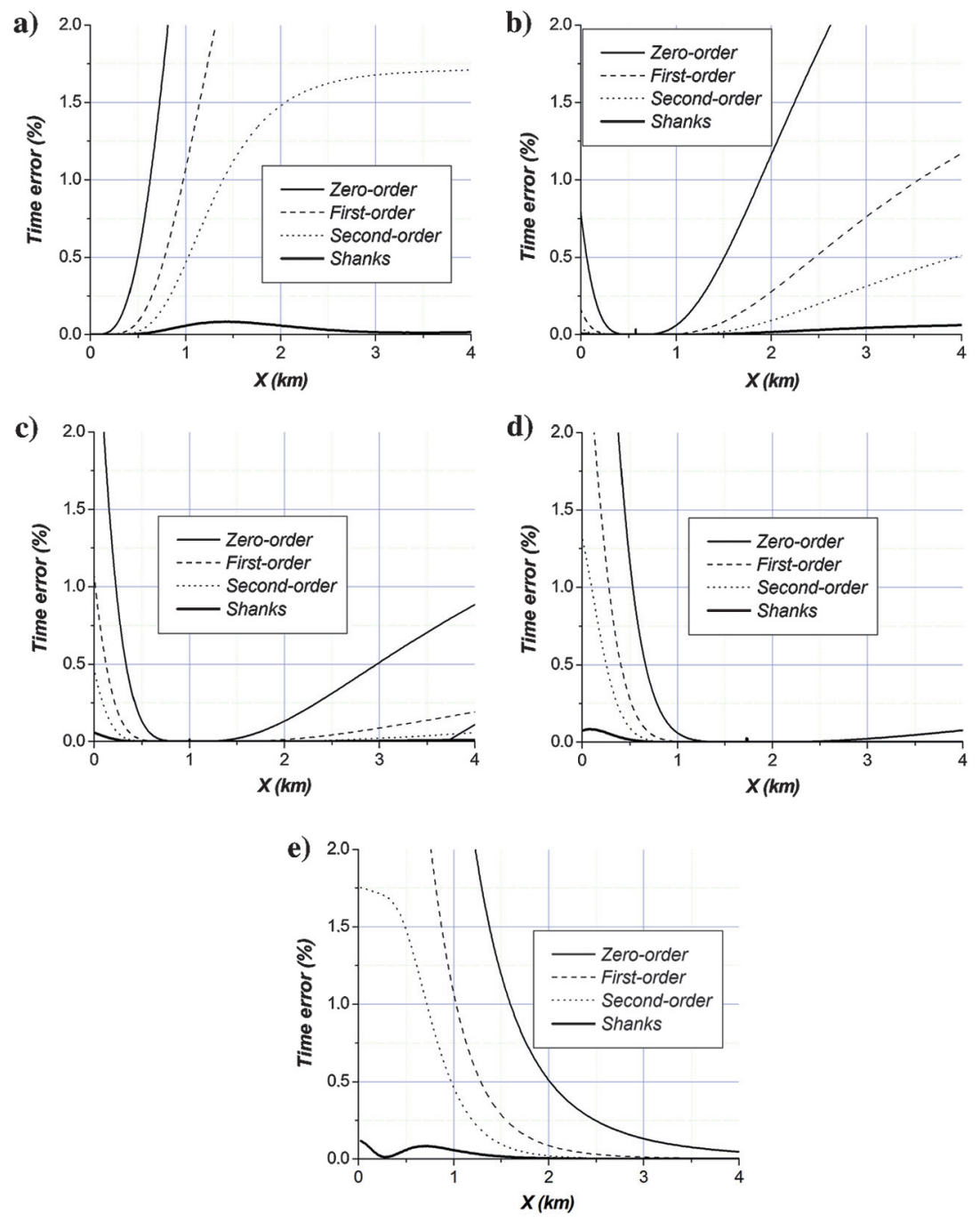

Figure 2. The relative error in traveltime from zero-, first-, and second-order expansions in eta and Shanks approximation for model two with symmetry angles: $0^{\circ}$ (top left), $30^{\circ}$ (top right), $45^{\circ}$ (middle left), $60^{\circ}$ (middle right), $90^{\circ}$ (bottom). 

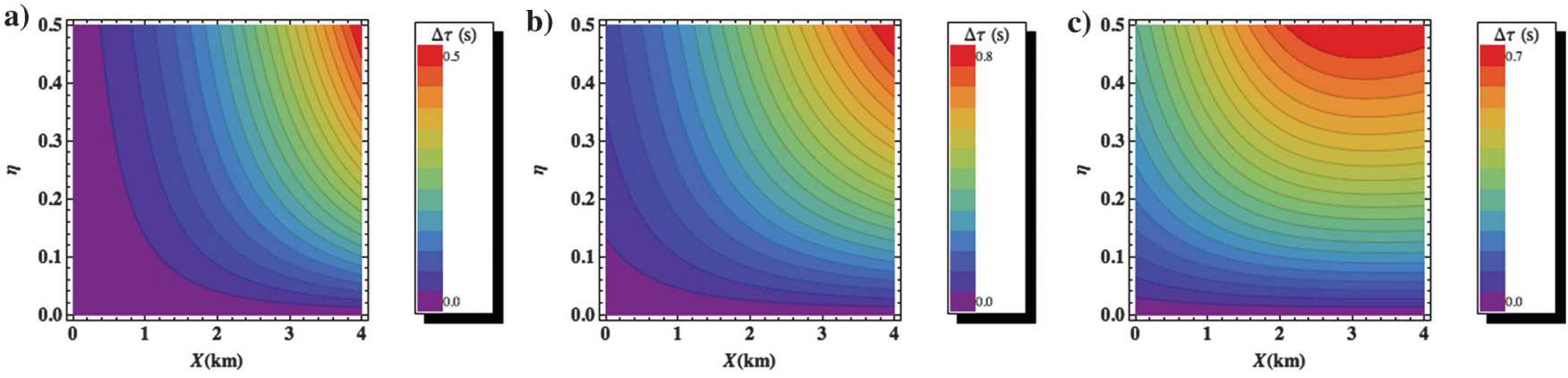

Figure 3. The traveltime difference from equations 4 and 6 computed for model two with three different tilts $20^{\circ}$ (a), $40^{\circ}$ (b), and $60^{\circ}$ (c)
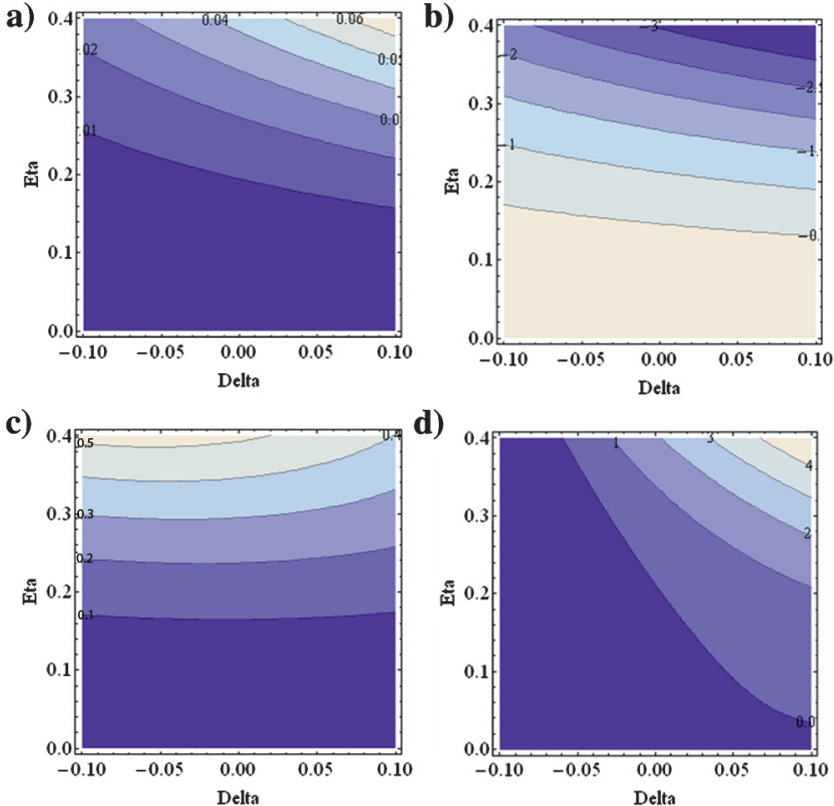

Figure 4. Relative errors (\%) in vertical traveltime (top) and in vertical NMO velocity (bottom) for a homogeneous TTI model with tilt of $20^{\circ}$ (left) and tilt of $40^{\circ}$ (right).

accurate homogeneous media traveltime representations that can be used for moveout analysis in TTI media.

\section{CONCLUSIONS}

Expanding the traveltime solutions of the TI eikonal equation in power series in terms of parameter $\eta$ provides an efficient tool to estimate (scan) $\eta$ in a generally inhomogeneous elliptically isotropic background medium. Using the Shanks transform, we managed to improve the accuracy of the expansion. For a homogeneous-tilted transversely isotropic medium, we obtain analytic nonhyperbolic moveout equations that are generally simple and yet accurate for large offsets. These equations reveal some of the properties of the traveltime dependency on $\eta$ and show that, with an increase in symmetry axis angle, the influence of $\eta$ is apparent even for short offsets.

\section{ACKNOWLEDGMENTS}

Alexey Stovas would like to acknowledge the ROSE project for financial support. Tariq Alkhalifah is grateful to KAUST for its support.

\section{REFERENCES}

Alkhalifah, T., 1998, Acoustic approximations for processing in transversely isotropic media: Geophysics, 63, 623-631, doi: 10.1190/1.1444361.

Alkhalifah, T., 2011a, Scanning anisotropy parameters in complex media: Geophysics, 76, no. 3, U13-U22, doi: 10.1190/1.3555040.

Alkhalifah, T., 2011b, Traveltime approximations for transversely isotropic media with an inhomogeneous background: Geophysics, 76, no. 3 , WA31-WA42, doi: 10.1190/1.3555040.

Alkhalifah, T., and P. Sava, 2010, A transversely isotropic medium with a tilted symmetry axis normal to the reflector: Geophysics, $\mathbf{7 5}$, no. 3, A19A24, doi: 10.1190/1.3409114.

Audebert, F. S., A. Pettenati, and V. Dirks, 2006, TTI anisotropic depth migration - Which tilt 358 estimate should we use?: 68th Annual International Conference and Exhibition, EAGE, Extended Abstracts, P185.

Bender, C. M., and S. A. Orszag, 1978, Advanced mathematical methods for scientists and engineers: McGraw-Hill.

Golikov, P., and A. Stovas, 2011, Traveltime parameters in a tilted elliptical anisotropic medium: 73rd Annual International Conference and Exhibition, EAGE, Extended Abstracts, P041.

Grechka, V., and A. Pech, 2006, Quartic reflection moveout in a weakly anisotropic dipping layer: Geophysics, 71, no. 1, D1-D13, doi: 10 $.1190 / 1.2159047$.

Huang, T., S. Xu, J. Wang, G. Ionescu, and M. Richardson, 2008, The benefit of TTI tomography for dual azimuth data in Gulf of Mexico: 78th Annual International Meeting, SEG, Expanded Abstracts, 27, 222-226.

Pech, A., I. Tsvankin, and V. Grechka, 2003, Quartic moveout coefficient: 3D description and application to tilted TI media: Geophysics, 68, 16001610, doi: 10.1190/1.1620634.

Sena, A. G., 1991, Seismic traveltime equations for azimutally anisotropic and isotropic media: Estimation of interval elastic properties: Geophysics, 56, 2090-2101, doi: 10.1190/1.1443021.

Zhou, H., D. Pham, S. Gray, and B. Wang, 2004, Tomographic velocity analysis in strongly anisotropic TTI media: 74th Annual International Meeting, SEG, Expanded Abstracts, 23, 2347-2350. 